\title{
4 Kommunikation in der Ausstellung: Theorie
}

Ziel des zweiten Untersuchungsteils ist die Rekonstruktion der sozialen Praxis des gemeinsamen Museumsbesuchs. Dazu werde ich untersuchen, wie die Besucher den Ausstellungsraum für ihren Museumsbesuch nutzen, wie sie das im Ausstellungsraum Arrangierte als Zeichen in einem Kommunikationsprozess interpretieren, wie sie dabei Zeichen aus den unterschiedlichsten Zeichenvorräten zueinander in Beziehung setzen und wie sie all das, was im Raum wahrnehmbar ist, für ihre lokale Konstruktion von geteiltem Wissen zu dem in der Ausstellung präsentierten Fachbereich nutzen. In der Zusammenschau wird sich so ein Bild der typischen Aktivitäten der Besucher ergeben, die die soziale Praxis des gemeinsamen Museumsbesuchs ausmachen.

Wie schon in der Darstellung der theoretischen Grundlagen der Kommunikation durch die Ausstellung in Kapitel 2 möchte ich auch hier zunächst Forschungsansätze vorstellen, die sich direkt mit dem Museumsbesuch befassen (4.1): Konkret handelt es sich um Arbeiten aus der musealen Besucherforschung, der Museumspädagogik und der musealen Lernforschung, die die ,Besuchserfahrung' empirisch zu vermessen suchen und der Frage nachgehen, wie die Besucher durch die Nutzung der Ausstellung Wissen erwerben können. Den Abschluss dieses Abschnitts bildet die Vorstellung einer Anzahl von Arbeiten, die unterschiedliche Aspekte des Museumsbesuchs aus einer gesprächsanalytischen Perspektive betrachten.

Diese schlagen die Brücke zu Abschnitt 4.2, in dem ich Forschungsansätze referiere, die sich mit der Raumgebundenheit von Kommunikation, der Multimodalität und der Kommunikation von Wissen beschäftigen - den Aspekten also, deren fundamentale Bedeutung für die Ausstellungskommunikation in Kapitel 1 herausgearbeitet worden ist und nach denen die Darstellung der theoretischen Anknüpfungspunkte in Kapitel 2 gegliedert ist. Allerdings treten diese Aspekte unter veränderten Vorzeichen in Erscheinung: die Raumgebundenheit nun unter dem Gesichtspunkt, wie der Raum und die im Raum arrangierten Objekte für die Interaktion relevant werden können, die Multimodalität nun mit Konzentration auf die an den Körper der Museumsbesucher gebundenen Ausdrucksressourcen und die Wissenskommunikation nun fokussiert auf die interaktiven Verfahren der lokalen Konstruktion von Wissen beim gemeinsamen Museumsbesuch. Und während in Kapitel 2 Textlinguistik und Semiotik die relevanten Bezugspunkte waren, nimmt nun die Konversationsanalyse diese Position ein. 


\subsection{Forschungen zur Kommunikation in der Ausstellung}

Interessiert man sich für die Museumsbesucher und ihre Nutzung der Ausstellung, bieten sich die empirische Besucherforschung, die Forschung zum musealen Lernen und die Museumspädagogik als relevante Anknüpfungspunkte an. ${ }^{1}$ Zwischen diesen unterschiedlichen Forschungsbereichen gibt es gewisse Unterschiede in der Schwerpunktsetzung. So sind die Besucherforschung und die museale Lernforschung konsequenter auf die empirische Fundierung ihrer Behauptungen ausgerichtet als die Museumspädagogik. Und in Museumspädagogik wie Besucherforschung ist der Optimierungsgedanke stärker als in der musealen Lernforschung, die sich vor allem als Grundlagenforschung versteht. Trotz diesen Unterschieden gibt es zahlreiche thematische, methodische sowie personelle Überschneidungen, weshalb die folgende Darstellung nicht primär nach der disziplinären Verortung der vorgestellten Arbeiten gegliedert ist, sondern entlang einer Reihe von Entwicklungen, die für die Erforschung der Kommunikation in der Ausstellung bedeutsam sind.

Die erste dieser Entwicklungen verläuft, wie Hooper-Greenhill (2008) beschreibt, von anekdotischen Studien zu solchen Untersuchungen, die auf das allgemeine Verstehen des Museumsbesuchs, der beim Besuch ablaufenden Prozesse und der sie bestimmenden Faktoren abzielen (2008: 362). Während viele der frühen Studien zur Besucherforschung lediglich für die auftraggebende Institution von Nutzen waren, bieten die Untersuchungen, die an den grundlegenden Bedingungen und Mechanismen des Museumsbesuchs interessiert sind, für die Erforschung der Kommunikation in der Ausstellung wichtige Einsichten.

Die zweite Entwicklung betrifft das Bild des Besuchers und seiner Aktivitäten im Museum. Anstelle den Besucher als bloßen Empfänger von Bedeutungen zu verstehen, sieht man ihn immer häufiger als aktiven Konstrukteur von Bedeutungen:

As long as successful communication was conceptualized as the effective transmission of a clearly defined message through an appropriate medium to a large mass of receivers, the focus on measuring the take-up of the message seemed to have the potential to lead to improved educational provision. (Hooper-Greenhill 2008: 371)

1 Einen Einblick in den aktuellen Stand der Besucherforschung geben Wegner (2019) und Anderson (2019); praktisch orientiert: Deutscher Museumsbund (2019); zur Museumspädagogik in Deutschland und ihrer Entwicklung: Grünewald Steiger (2016). Roskam (2020) illustriert den fachdidaktischen Blick auf Naturwissenschaftsmuseen. 
Nicht mehr das ,Aufnehmen“ der Botschaft steht seit der Jahrhundertwende im Blickpunkt, sondern die eigenständige Konstruktion von Bedeutungen in sozialen Aushandlungsprozessen. Diese neue Sicht des Besuchers lässt sich gut in die konversationsanalytisch inspirierte Untersuchung der Kommunikation in der Ausstellung anschließen, die ja die Konstruktionsleistungen der Interaktionsteilnehmer betont.

Eine dritte Entwicklung ist die Zunahme von Beobachtungsstudien, die sich allerdings noch nicht gegen die dominierenden Fragebogen- und Interviewstudien durchgesetzt haben (Fu et al. 2019). Diese bringen aber das generelle Problem mit sich, dass sich die Forschenden auf Selbstauskünfte der Besucher verlassen müssen. Das gilt auch für Mischformen, in denen der per Video dokumentierte Besuch nur als Input für ein „in-situ interview“ genutzt wird (Schmitt 2016). Hinzu kommt, dass diese Selbstauskünfte, gerade wenn sie erst am Ende des Besuchs erhoben werden, häufig wenig präzise sind. Fragen, die für das Verstehen der Kommunikation in der Ausstellung fundamental sind - etwa wie die Besucher einzelne Objekte oder einzelne Texte genutzt haben, über welche Aspekte dieser Objekte oder Texte sie mit ihren Begleitern gesprochen haben oder welche Strategien sie gemeinsam genutzt haben, um sich die Bedeutung eines Exponats zu erschließen - lassen sich so schwerlich erforschen. Museumsstudien, die mit Beobachtung arbeiten (sei sie offen oder verdeckt), sind bis auf den heutigen Tag seltener anzutreffen. ${ }^{2}$ Das Spektrum dieser Studien reicht von ethnographischen Untersuchungen (klassisch: Véron/Levasseur 1991) bis hin zu Versuchsanordnungen, die die ästhetische Erfahrung beim Museumsgang zu vermessen suchen, indem sie Besucherbewegungen tracken (Pierroux/Steier 2016) oder Museumsbesucher mit einem Datenhandschuh ausrüsten, der dem Computer ständig genaue Positionsangaben des Besuchers sendet und sie mit physiologischen Daten verknüpft (Tröndle 2008, Tröndle et al. 2014). Diesen Studien ist gemeinsam, dass sie nicht die räumliche Orientierung der Besucher erfassen, sondern nur deren Position und Bewegung im Raum. Für die Untersuchung der Bedeutungskonstitution im Rahmen des Museumsbesuchs ist das nicht feinkörnig genug. Zudem arbeiten viele quantitativ arbeitende Beobachtungsstudien mit so starken Vereinfachungen und Abstraktionen, dass sie nur noch schwer auf die Museumserfahrung zu beziehen sind, die es zu beschreiben gilt, etwa wenn sie lediglich registrieren, wie lange Besucher vor einem Exponat verweilen, um dann

2 Serrell (2020) verweist zwar darauf, das ein großer Prozentsatz von Museen Beobachtungen zum Besucherverhalten anstellen. Ihre Überblickstudie zeigt jedoch, dass damit oft nur das Festhalten minimaler Beobachtungsdaten (im Zentrum: Verweildauer) gemeint ist. 
einen Wert wie den „sweep rate index“ $z u$ berechnen (durchschnittliche Verweilzeit geteilt durch die Ausstellungsfläche, s. Serrell 2020). ${ }^{3}$

Eine weitere Entwicklung in der Museumsforschung, die sich für die Erforschung der Kommunikation in der Ausstellung nutzen lässt, ist das Aufkommen einer Forschung zum musealen Lernen, die sich aus unterschiedlichen Disziplinen und Ansätzen speist (s. Schwan/Grajal/Lewalter 2014, Noschka-Roos/Lewalter 2016). Ihr verdankt sich

- zuallererst die Entdeckung des Museums als eines „Lernorts“ in den 1970er Jahren (Spickernagel/Walbe 1976, Englert/Kiupel 2012, Grünewald Steiger 2016);

- die Beschreibung des Museumsbesuchs als einer Form des „informellen Lernens“ (Schrader/Stadler/Körber 2008: 100; Hauan/Kolstø 2014, Allen/ Petermann 2019) oder

- die Definition von „learning outcomes“, die mehr als nur den Erwerb deklarativen Wissens umfassen, sondern auch „[c]hange in attitudes or values“, „enjoyment, inspiration and creativity“ usw. (Hooper-Greenhill 2002: 11-17, National Research Council 2009: 27-33).

Für die Untersuchung der Kommunikation in der Ausstellung sind besonders eine Anzahl von Arbeiten von Interesse, die Lernen über die zunehmende ,konversationelle Elaborierung“ zu erfassen versuchen (s. Leinhardt/Crowley 1998, Leinhardt/Crowley/Knutson 2002 oder Leinhardt/Knutson 2004). Leinhardt/Crowley (1998) beispielsweise nehmen Gespräche von Besuchern während des Museumsbesuchs auf und vergleichen sie mit Interviews vor und nach dem Besuch. Lernen zeigt sich für die Autoren darin, dass die Besucher das im Museum behandelte Thema nach dem Besuch gründlicher oder detailreicher besprechen und einzelne Informationen besser in einen Gesamtrahmen integrieren können als zuvor. Deshalb gehen Arbeiten aus diesem Ansatz nicht nur auf den genauen Wortlaut der Besucheräußerungen ein, sondern bisweilen sogar auf paraverbale Signale (etwa den Wechsel vom ,Lehrerton' zu einem informelleren Sprachduktus: Crowley/Jacobs 2002). Von der Untersuchung der Gespräche erhoffen sich die Vertreter dieses Ansatzes Einblicke in „the subtle, moment-by-moment learning that characterizes learning in museums“ (Allen 2002: 260). Doch widmet sich nur ein kleinerer Teil der Studien den hier interessierenden Mikrophänomenen, etwa dem sukzessiven Aufbau eines Verständnisses einzelner Exponate. Die Grundeinheit der Analyse ist häufig der vollständige Museums- oder Ausstellungsbesuch.

3 Zur Kritik an dieser Art von Besucherforschung s.a. Heath/vom Lehn/Osborne (2005: 95-98). 
Schließlich ist innerhalb von Museumspädagogik, Besucherforschung und musealer Lernforschung die Entwicklung zu beobachten, dass das Lernen im Museum zunehmend als soziales Handeln verstanden wird. ${ }^{4}$ Lernen ist aus dieser Sicht „socially and culturally constructed through people's actions within a specific community of practice“ (Ellenbogen/Luke/Dierking 2007: 19). Zum einen heißt das, dass zur Analyse des Lernens im Museum auch die Rolle von früheren Erfahrungen, von gemeinsamen Werten der „community of practice“ usw. berücksichtigt werden müssen. Zum anderen wird der Blick auf die Verbindungen zwischen dem Museumsbesuch einerseits und Praktiken der Identitäts- und Bedeutungskonstruktion in relevanten sozialen Gruppen geföffnet. Zwei Ansätze haben diese Entwicklung vorangetrieben. Zum einen Arbeiten, die sich auf Vygotsky beziehen und die Rolle der Interaktion für das Lernen betonen (z.B. Leinhardt/Crowley/ Knutson 2002) zum anderen Arbeiten aus dem Umfeld des „Contextual Model of Learning“ (z.B. Falk/Dierking 2000, Falk/Storksdieck 2005, Harms/Krombaß 2008, Falk/Dierking 2013). Nach Falk/Storksdieck (2005) gilt es, folgenden Kontexten Rechnung zu tragen: einem ,persönlichen Kontext‘, zu dem beispielsweise die Besuchsmotivation gehört, aber auch Vorwissen und Vorerfahrungen, einem ,soziolkulturellen Kontext' sowie einem ,physical context‘. Zu Letzterem zählt nicht nur der Ausstellungsraum und das in ihm Wahrnehmbare, sondern auch „[s]ubsequent reinforcing events and experiences outside the museum“ (2005: 747).

Der programmatische Einbezug des personalen und soziokulturellen Kontexts hat zu einer beträchtlichen Steigerung der Datenvielfalt geführt. (Ellenbogen/Luke/Dierking 2007) nennt beispielsweise

video and audio recording of moment-by-moment interactions; pre-, post-, and post-postinterviewing; journaling; and talk-aloud cued visits; and [...] providing family members with cameras as a documentation and meaning-making tool [...]. (ebd.: 20f.)

Die Art und Weise, wie die an sich richtige Einsicht, dass Museumsbesuche in soziale Kontexte eingebunden sind, in der Forschung umgesetzt worden ist, scheint mir in zweifacher Hinsicht problematisch zu sein. Zum einen geht die Berücksichtigung des personalen und soziokulturellen Kontexts auf Kosten der gründlichen Analyse dessen, was die Besucher im Ausstellungsraum wahrnehmen oder lesen können. Ohne eine Bezugnahme auf das multimodale kommunikative Angebot im Ausstellungsraum kann die Analyse den Besonderheiten

4 Parallel dazu gibt es auch Studien, die das Lernen im Museum aus kognitiv-motivationaler Perspektive in den Blick nehmen: z.B. Noschka-Roos/Lewalter (2013). 
der Ausstellungskommunikation aber nicht gerecht werden. Zum anderen ist aus der Entdeckung der sozialen Natur des Museumslernens häufig die Notwendigkeit abgeleitet worden, mehr und andere Daten als die Gesprächsaufnahmen zur Grundlage der Analyse zu machen (etwa indem man die Vorerfahrungen der Besucher per Fragebogen erfasst oder ihr soziales Umfeld ethnographisch ausleuchtet, ja indem man sogar Nicht-Besucher in den Blick nimmt: Renz 2016). Dagegen wird hier die Ansicht vertreten, dass die Analyse sich auf die Gesprächsdaten allein beschränken kann, ohne fürchten zu müssen, die sozialen Kontexte, die für die Praxis des gemeinsamen Museumsbesuchs relevant sind, nicht ausreichend berücksichtigen zu können. Grundlage ist das sogenannte display-Prinzip der Konversationsanalyse (Mondada 2011b: 542f.). Deppermann (2008: 50) beschreibt dieses Prinzip so:

Die Konversationsanalyse geht von der zentralen methodologischen Prämisse aus, daß Gesprächsteilnehmer einander aufzeigen (,display‘, z.B. Schegloff 1997), welchen Sinn und welche Bedeutsamkeit sie ihren Äußerungen [und allgemein: ihren Handlungen, W.K.] wechselseitig zuschreiben. Wäre dies nicht wenigstens teilweise und immer wieder so, wären Handlungskoordination und Verständigung unmöglich.

Für die Kontexte des Museumsbesuchs heißt das: Die Besucher machen sich alle soziokulturellen Zuordnungen und Wertesysteme, die für ihre soziale Praxis des gemeinsamen Museumsbesuchs relevant sind, sichtbar und hörbar. Um das Interaktionsgeschehen vollständig verstehbar zu machen, ist es also nicht nötig, auf zusätzliche Informationen, von außen` zurückzugreifen. Der Sinn des Interaktionsgeschehens kann ,von innen heraus‘ entwickelt werden, d.h. auf der Grundlage von Gesprächsdaten, die ,a naturally occurring and meaningful process and product of the museum experience“ sind (Allen 2002: 260).

Viele Studien lösen die Gesprächsaufnahmen im Laufder Analysen in isolierte Sinneinheiten auf, die sie unterschiedlichen Codes (im sozialwissenschaftlichen, nicht semiotischen Sinn) zuordnen. Damit wird allerdings der Gesprächszusammenhang zerstört, und die Beschäftigung der Besucher mit der Ausstellung kann nicht mehr in ihrem dynamischen Verlaufs untersucht werden. In den letzten Jahren gibt es aber vermehrt Arbeiten, die den Gesprächszusammenhang erhalten, um so dem dynamischen Interaktionsgeschehen im Museum auf die Spur zu kommen. Hierzu zählen Arbeiten aus dem Kontext der Science Education, etwa Ash (2002 oder 2007) oder (Kelly 2007), die die Dynamik der Bedeutungskonstruktion und das wissenschaftliche Argumentieren und Schlussfolgern bei der Nutzung von Museumsausstellungen detailliert erforschen.

Auch Nettkes Studie zu Führungen im Naturkundemuseum (Nettke 2009) analysiert die Konstruktion von Wissen im Gesprächszusammenhang. Nettkes Untersuchung analysiert die Führung als Interaktion zwischen Führenden und 
Geführten. Gestützt auf die Methoden der Gesprächsforschung und ausgehend von Aufzeichnungen authentischer Führungen identifiziert Nettke wiederkehrende Handlungsmuster, die zusammen die Typik der Museumsführung ausmachen. Innerhalb der großräumigen „Komponenten des Handlungsschemas“ beschreibt Nettke jeweils häufig ausgeführte „Kernaktivitäten“ und deren relevante „Arbeitsschritte“. Besonders innovativ ist, dass der Autor bei seiner Analyse der Vermittlungsaktivitäten nicht nur die inhaltliche Vermittlung von Wissen berücksichtigt (Nettke spricht von „Themaarbeit“), sondern auch die Herstellung der räumlichen Voraussetzungen der Vermittlung (die „Raumarbeit“, 2009: 376). Letztere definiert der Autor so:

Bei der Raumarbeit geht es um die Koordination der Sinneswahrnehmung auf Objekte sowie damit verbunden die Koordination der Körperposition der Akteure im Raum. Zudem werden unter Raumarbeit auch Praktiken zur Koordination der Fortbewegung der Akteure im Raum subsumiert. In der Sprache des Feldes wird insbesondere die Raumarbeit mit Aktivitäten wie ,Zeigen` und ,Führen` bezeichnet. (2009: 421-441)

Hier berühren sich die Forschungen aus dem Museumskontext mit der konversationsanalytisch oder ethnomethodologisch inspirierten Forschung zum Museum, die ihre Wurzeln bald in der interaktionalen Soziologie, bald in der linguistischen Gesprächsforschung hat.

$\mathrm{Zu}$ nennen sind hier speziell die soziologischen Arbeiten von Dirk vom Lehn, Christian Heath und ihren Londoner Kollegen am Kings' College, die in über zehnjähriger Forschung viele unterschiedliche Aspekte der Interaktion im Ausstellungsraum beleuchtet haben. Während es in der hier vorgestellten Untersuchung nur um ,traditionelle“ naturwissenschaftliche Museen geht, untersucht die Londoner Forschergruppe ebenso Besuche in Kunstmuseen (siehe dazu besonders Heath et al. 2002, Heath/vom Lehn 2004 oder vom Lehn/Heath 2007, vom Lehn 2013, Lehn/Heath 2016) oder in ,interaktiven' Science Centres (dazu z.B. vom Lehn/Heath/Hindmarsh 2001, Heath/vom Lehn/Osborne 2005). Ihre zentrale Erkenntnis ist, dass der Museumsbesuch als soziale Interaktion untersucht werden muss, und nicht mit Rückgriff auf Merkmale des Raums und der Exponate oder mit Rückgriff auf Mechanismen der menschlichen Wahrnehmung und Kognition erklärt werden kann. Das folgende Zitat fasst wesentliche Aspekte dieser zentralen Erkenntnis zusammen:

An individual's experience of objects and artefacts in museums and galleries may not simply derive from the cognitive or psychological dispositions or abilities that he or she brings to bear when looking at an exhibit, nor necessarily the knowledge a person may have of particular forms of art, artefact and the like. Their investigation and experience of the exhibit may emerge, then and there, through their interaction with others, both those they are with and those who happen to be within the same space. Their knowledge and understanding 


\begin{abstract}
of particular forms of art and artefact, their cognitive and psychological abilities have to be deployed with regard to the contingencies at hand at any particular moment, in their interaction with others. The interaction does not so much permeate a set of pre-established dispositions or bodies of knowledge, but rather provides the material and interactional circumstances through which people come to see and understand exhibits in particular ways. The 'situated' or 'occasioned' conduct and experience of visitors are hardly reducible to cognitive abilities and dispositions of the participants; rather conduct and experience emerge in and through socially organized actions and interaction. What is noticed, seen, inspected, reviewed, discussed and experienced arises from within interaction and is contingent upon the occasioned conduct of the participants themselves. (Heath/vom Lehn 2004: 59)
\end{abstract}

Als Ergebnis der Interaktion der Museumsbesucher zu verstehen ist zum einen die Nutzung des Ausstellungsraums durch die Besucher, zum anderen auch die Bedeutung der Exponate und die Art der Erfahrungen, die mit deren Hilfe gewonnen werden können. So zeigen vom Lehn, Heath und Kollegen auf der Grundlage der detaillierten Untersuchung von Einzelfällen, wie die ,Navigation' durch den Ausstellungsraum von den Besuchern interaktiv ausgehandelt wird, wie die Annäherung an ein Ausstellungsstück (vom Lehn/Heath 2007), die Möglichkeiten der Betrachtung oder Manipulation des Exponats (speziell zur Aushandlung der Beteiligung an der Nutzung, interaktiver‘ Exponate: Heath/vom Lehn/ Osborne 2005) oder die Abwendung von dem Ausstellungsstück durch spezielle, fein aufeinander abgestimmte körperliche wie sprachliche Aktivitäten der Besucher geleistet werden.

Die Aktivitäten, mit denen die Besucher ihre gemeinsame Bewegung durch den Museumsraum hervorbringen, sind - wie vom Lehn (2013) am Prozess der Abwendung von einem Exponat zeigt - sequenziell geordnet: Auf eine, Einladung zur Abwendung‘ als ,erste Handlung، folgt als ,zweite Handlung‘ ein ,Annehmen der Einladung' oder ein ,Ablehnen', das zu einer Expansion der Abwendungssequenz führen kann, sprich: zu weiteren Aktivitäten, die auf eine gemeinsame Abwendung, ein „concerted onward movement“ (ebd.: 85) hinarbeiten. Interaktiv hergestellt wird auch - und das mag auf den ersten Blick weit weniger einleuchten - die Bedeutung der Exponate und die Erfahrungen, die an ihnen zu machen sind. Das beginnt, wie (vom Lehn/Heath 2007: 11) exemplarisch zeigen, schon damit, dass die Position und die Blicke des anderen so gelenkt werden, dass ein behauptetes Merkmal des Exponats gesehen werden kann, und es endet mit dem Hervorbringen von Phänomenen für die Wahrnehmung durch den jeweils anderen Besucher (etwa durch die Bedienung eines ,interaktiven' Exponats in einem Science Centre, Heath/vom Lehn 2001). Die Merkmale der Exponate sind nicht einfach ,da', sie werden durch das, was die Besucher sich sprachlich oder gestisch aufzeigen, erst hervorgebracht. Besonders deutlich wird das, wenn die Merkmale, um die es geht, kaum oder gar nicht sichtbar sind, aber durch die 
Gesten eines der Besucher für den anderen sichtbar gemacht werden (Heath/ vom Lehn 2004: 49-53). Die Besucher ,konfigurieren‘ also die Ausstellungsstücke für ihre Mitbesucher, indem sie diesen Merkmale zuschreiben und mit körperlich-gestischen Mitteln dafür sorgen, dass jene die betreffenden Merkmale auch wahrnehmen können. ${ }^{5}$ Im Zuge dieser ,Konfiguration“ der Ausstellungsstücke können die Exponate bisweilen durch die Besucher lebendig gemacht werden, indem Merkmale des Exponats durch den Körper eines der Besucher dargestellt werden. So können die anderen Besucher die relevanten Merkmale des Exponats nicht mehr nur aus der Beschreibung erschließen, sondern gleichsam direkt ,als Zeugen` erfahren (Hindmarsh/Heath 2003). Zu dieser ,Konfiguration‘ gehört häufig auch, dass die Besucher die ästhetischen Erfahrungen und emotionalen Reaktionen anzeigen, die das Exponat im anderen erwecken soll: Begeisterung, Ehrfurcht, Freude, Ablehnung, Ekel usw. Aus all diesen Beobachtungen schließen die Autoren, dass sowohl Lernen als auch ästhetische Erfahrungen durch Exponate keinesfalls allein aus Eigenschaften der Exponate oder des Ausstellungsraums ableitbar wären, sondern sich nur als Resultat der Interaktion der Besucher im Ausstellungsraum verstehen lassen:

[T] he participants' experience is inseparable from and permeated with the ways in which others have attempted to configure the object and the ways of seeing; the exhibit is discovered, enlivened, perceived, and assessed in, and through, the interaction of the participants. (Heath/vom Lehn 2001: 292)

Ganz ähnliche Themen untersucht die konversationsanalytische Forschung zur Interaktion in Ausstellungen, die ihre Wurzeln in der Linguistik hat. Die linguistische Beschäftigung mit dem Museumsbesuch erwächst zum einen aus dem Interesse, der Räumlichkeit von Interaktion auf den Grund zu gehen. Zum einen geht es um die Frage, wie die Museumsbesucher einen gemeinsamen Interaktionsraum aufbauen, wobei die Betonung bald auf der ,Choreographie‘ der sprachlichen und körperlich-gestischen Beiträge liegt, mit denen der museumsspezifische Interaktionsraum hervorgebracht wird (Kesselheim 2012, Hausendorf 2010 mit einem Abschnitt zur Ausstellungskommunikation), bald zusätzlich auf der komplexen internen Strukturierung des Interaktionsraums und den unterschiedlichen Formen abgestufter Beteiligung an der Museumsinteraktion (Pitsch 2012). Dabei gerät nicht nur der durch die Aktivitäten der Besucher hervorgebrachte

5 Auch zeigen sie ihren Mitbesuchern, wie jene die multimodalen Ressourcen im Ausstellungsraum miteinander verbinden sollen: „Through their accomplishments visitors interweave and intermingle exhibits and their labels“ (Heath/vom Lehn 2001: 293). Leider gehen die Autoren diesem Aspekt nicht weiter nach. 
Interaktionsraum in den Blick, sondern auch der vorstrukturierte Ausstellungsraum mit seinem multimodalen Kommunikationsangebot. In diesem Zusammenhang widmen sich Kesselheim (2012) und Hausendorf/Kesselheim (2016) der Frage, wie die Besucher Elemente im Ausstellungsraum als Zeichen behandeln, die sie als Teil eines Kommunikationsangebots nutzen (sicht- und hörbar z.B. im lauten Vorlesen von Objektkennungen), und Pitsch (2012) geht Prozessen nach, durch die Objekte im Ausstellungsraum den Status von Exponaten erhalten - oder ihn auch wieder verlieren. Der interaktiven Herstellung von Wissen und körperlichen Erfahrungen widmen sich schließlich Kesselheim (2010a) und Kesselheim/Brandenberger (2021).

Trotz ihrer unterschiedlichen disziplinären Verortung sind die hier dargestellten soziologischen und linguistischen Untersuchungen zur Interaktion im Museum vollständig kompatibel und gewähren sich ergänzende Einsichten in die interaktive ,Mechanik‘ des Museumsbesuchs.

\subsection{Konversationsanalytische Anknüpfungspunkte}

Bis hierhin sind solche Forschungsansätze und -arbeiten dargestellt worden, die sich direkt mit der Kommunikation in der Ausstellung auseinandergesetzt haben. Im restlichen Kapitel geht es um Forschungen aus der linguistischen Gesprächsforschung und benachbarten Disziplinen, die sich denjenigen Aspekten von Interaktion widmen, die wir weiter oben (1.2) als die grundlegenden Merkmale der Ausstellungskommunikation identifiziert haben: die Raumgebundenheit von Interaktion und ihre interaktive Hervorbringung sowie die lokale Genese von Wissen in der Interaktion.

Anders als in Kapitel 2 können diese drei Aspekte allerdings nicht in je einem Unterabschnitt vorgestellt werden. Während sich die gesprächsanalytische Beschäftigung mit der interaktiven Konstruktion von Wissen noch problemlos als eigenständiger Themenstrang isolieren lässt (s.u. 4.2.5), sind der Raum- und der Multimodalitätsaspekt in der konversationsanalytischen Forschungspraxis derart eng miteinander verflochten, dass mir eine separate Darstellung als nicht gangbar erscheint: Raum ist nämlich in der Konversationsanalyse bisher überwiegend als etwas untersucht worden, das durch multimodale Aktivitäten der Interaktionsbeteiligten hervorgebracht wird. Stattdessen werde ich zunächst das konversationsanalytische Verständnis des Raums und seiner Rolle in der Interaktion darstellen (4.2.1), dann werde ich auf die Frage eingehen, wie die Interaktionsteilnehmer mit Hilfe multimodaler, an ihre Körper gebundener Ausdrucksressourcen (also Gestik, Bewegungen durch den Raum usw.) die räumlichen Voraussetzungen für ihre Interaktion, also den „Interaktionsraum“, 
schaffen (4.2.2). Abschnitt 4.2.3 und 4.2.4 referieren konversationsanalytische Arbeiten zur multimodalen Herstellung bzw. Aktivierung des Raums durch die Interaktionsbeteiligten, wobei Abschnitt 4.2.4 gesondert die Frage aufgreift, wie das in der Interaktionssituation Vorgefundene, also beispielsweise der gebaute Raum und seine Objekte in eine konversationsanalytische Analyse einbezogen werden kann. Den Abschluss dieses Theoriekapitels bildet Abschnitt 4.2.5, der sich mit dem Aspekt der Wissenskommunikation beschäftigt.

\subsubsection{Das Raumkonzept der Konversationsanalyse}

Wie in Kapitel 1 erläutert, werde ich die Kommunikation durch die Ausstellung mit den Mitteln der Konversationsanalyse untersuchen (s. als Einführung ten Have 2007, Gülich/Mondada/Furchner 2008, Birkner et al. 2020). Diese hat nicht nur eine lange Erfahrung mit der Untersuchung von Interaktionsprozessen und verfügt daher über eine breite Palette erprobter Untersuchungsmethoden und -kategorien, die zur Erforschung der sozialen Praxis des gemeinsamen Museumsbesuchs genutzt werden können. Sie hat sich auch in der letzten Zeit entschieden der Multimodalität und Räumlichkeit der Interaktion zugewandt, die für die Kommunikation in der Ausstellung zentral sind.

Der Rückgriff auf die Konversationsanalyse bringt eine Reihe von Grundannahmen mit sich (s. Schenkein 1978: 1 zur „analytic mentality“ der Konversationsanalyse, s.a. Gülich/Mondada/Furchner 2008: 16-19), von denen ich hier nur einige aufgreifen möchte, die für den konversationsanalytischen Umgang mit dem Raum besonders folgenreich sind:

- Die soziale Wirklichkeit wird in all ihren Aspekten als Konstruktion verstanden.

- Diese Konstruktion geschieht im Vollzug der Interaktion durch die Aktivitäten der Interaktionsbeteiligten: Sie ist daher interaktiv (oft ist im Englischen von ,co-construction' die Rede, um diese spezifische Vorstellung von der Konstruktion zu betonen), und sie ist grundlegend an die Sequenzialität der Interaktion gebunden. Konversationsanalytische Untersuchungen interessieren sich für die Aktivitäten, mit denen die Interaktionsteilnehmer diese Hervorbringung zu Wege bringen und deren präzise sequenzielle Organisation.

- Aus dem Interesse an der Hervorbringungsleistung der Beteiligten erwächst auch die Forderung, die Perspektive der Interaktionsbeteiligten einzunehmen. Die konversationsanalytische Untersuchung fragt nicht nach einer von außen feststellbaren Logik oder Struktur der Daten. Stattdessen möchte sie 
wissen, wie die Teilnehmer die Welt ordnen, welches die Alltagsmethoden sind, mit denen sie ihre Interaktion organisieren.

Diese grundsätzliche Sicht der sozialen Wirklichkeit bestimmt auch das Raumkonzept der Konversationsanalyse, das ich nun darstellen und in den Kontext ähnlicher Raumkonzepte in anderen Disziplinen einordnen möchte.

Die Konversationsanalyse bezieht Stellung gegen ein physikalisches Konzept des geometrischen Raums, nach dem Raum nichts anderes ist als eine homogene und ,isotrope“ Ausdehnung (d.h. eine Ausdehnung, die sich in alle Richtungen gleich erstreckt), die man mit Hilfe von Werten in einem dreidimensionalen Koordinatensystem hinreichend beschreiben kann. Dieses geometrische Raumkonzept wird oftmals mit der so genannten „Container“-Vorstellung des Raums gleichgesetzt. Nach dieser Vorstellung ist der Raum so etwas wie ein allumfassendes Behältnis, in dem jede menschliche Handlung, jede Interaktion stattfindet. Der statische Container-Raum bestimmt als externer Faktor die Wahrnehmungs-, Bewegungs- und Handlungsmöglichkeiten der Interaktionsteilnehmer und wird von den Handlungen und Interaktionen, die in ihm ablaufen, nicht beeinflusst. Gegen diese „,Bucket“ theory of space“ (Drew/Heritage 1992: 19) setzt die Konversationsanalyse ein Raumkonzept, das mit den konversationsanalytischen Grundannahmen zum Herstellungscharakter der Wirklichkeit kompatibel ist und das Raum als etwas vom Menschen im Verlauf der Interaktion Konstruiertes und mit Sinn Versehenes in den Blick nimmt. Diese Auffassung ist nicht auf die Konversationsanalyse beschränkt, sondern wurde unter den unterschiedlichsten Bezeichnungen in verschiedenen Disziplinen und Forschungsansätzen diskutiert, und das nicht erst seit dem „spatial turn“ (s. etwa Döring/Thielmann 2008, Dünne/ Günzel 2006, speziell Dünne 2006) in den Geisteswissenschaften. Sie findet sich wieder, mit unterschiedlichen Schwerpunktsetzungen und theoretischer Begründung,

- in der breit rezipierten Unterscheidung von „space“ und „place“ (Tuan 1977), wo mit „space“ der physisch-materielle, mit Angaben geografischer Koordinaten definierbare oder mit geometrischen Mitteln beschreibbare Raum gemeint ist, während „place“ den sozial konstituierten Raum meint, dem durch menschliche Aktivitäten ein Sinn gegeben wird;

- in de Certeaus Unterscheidung von „Ort“ vs. „Raum“: Der „Ort“ ist bei ihm der geometrische Raum, der „Raum“ dagegen ist „ein Ort, mit dem man etwas macht“, d.h. der durch die Praktiken oder durch die Interpretationen seiner ,Nutzer‘ eine Bedeutung erhält (Certeau 1988: 218); ${ }^{6}$

6 De Certeaus Begriffe stehen der vorherrschenden Verwendung von „Raum“ und „Ort“ genau 
- mit strukturalistischem Hintergrund in Greimas' Unterscheidung von „étendue“ (der Raum-Substanz) und „espace“ (der Raum-Form, Greimas/ Courtés 1979) oder,

- auf Konstruktionsvorgänge im Individuum bezogen, in der (gestalt-)psychologischen Unterscheidung von „geographischer Umwelt“ und „Verhaltensumwelt“ oder „erlebter Umwelt“ (bei Koffka 1935).

Die Konversationsanalyse beschränkt sich nun nicht darauf, die physikalische Raumauffassung zu kritisieren. Vielmehr hat sie ein Raum-Konzept entwickelt, das mit den oben erwähnten konversationsanalytischen Grundannahmen kompatibel ist und eine genuin konversationsanalytische Raum-Analyse ermöglicht. Folgende Punkte charakterisieren den konversationsanalytischen Zugang zum Raum:

- $\quad$ Raum wird hergestellt. ${ }^{7}$ Dies geschieht in der Interaktion, und zwar durch sequenziell geordnete Hervorbringungsaktivitäten der Interaktionsbeteiligten, die es in der konversationsanalytischen Untersuchung zu rekonstruieren gilt.

- Die Konstruktion des Raums orientiert sich an den je aktuellen Bedürfnissen und Zielen, die die Teilnehmer in ihrer Interaktion verfolgen und wird kontinuierlich an deren Veränderungen im Fortgang der Interaktion angepasst: Raum wird ,acomplished, maintained and dynamically shaped in and for a particular interaction“ (Broth 2009).

- Raum wird als reflexive Figur gedacht: Raum wird von den Teilnehmern in der Interaktion hergestellt, aber gleichzeitig trägt er dazu bei, die Interaktion zu formen und ihr einen bestimmten Sinn zu verleihen (z.B. indem er ein Stehenbleiben im Raum zu einem Stehenbleiben vor einem Exponat macht, s.u. 6). Raum ist also „both action-shaping and action-shaped“ (Mondada 2009b: 3).

- Nötig ist es schließlich, Raum als Teilnehmerkategorie (vgl. ten Have 2007: 34f.) aufzufassen und zu rekonstruieren, wie Teilnehmer den Raum interpretieren und ihn in ihrer Interaktion nutzen.

entgegen. Bei de Certeau ist „Raum“ der anthropologische Raum (allgemein: „place“, übersetzt als „Ort“), und „Ort“ der physikalische (allgemein: „space“, dt. „Raum“).

7 Mit „Herstellung“ ist nicht nur die materielle Erzeugung räumlicher Strukturen gemeint. „Hergestellt“ wird der Raum nicht nur, indem Wände eingezogen, Stelltafeln aufgestellt und Texte angebracht werden, sondern auch, wenn der Raum als ein bestimmter Raum interpretiert wird (also beispielsweise: als „Ausstellungsraum“) oder Bereiche des Raums für eine bestimmte Nutzung aktiviert werden (beispielsweise: ein Raumbereich wird als „Durchgang“ verstanden und genutzt). 


\subsubsection{Die Herstellung des Interaktionsraums}

Eine große Zahl von Arbeiten hat in den letzten Jahren belegt, dass dieses Raumverständnis für die Analyse des sogenannten „Interaktionsraums“ fruchtbar gemacht werden kann. Der Interaktionsraum ist der Raum, den die Interaktionsbeteiligten für die Durchführung ihrer gemeinsamen Aktivität herstellen. Viele der Studien zum Interaktionsraum beziehen sich auf die Forschung der sogenannten „context analysis“ (Ciolek/Kendon 1980, Kendon 1990a), die früher noch als konversationsanalytische Arbeiten die Rolle von Blicken, Gesten und speziell der Körperausrichtung der Interaktionsbeteiligten für die Herstellung des gemeinsamen Interaktionsraums beschrieben hat.

Ciolek/Kendon (1980) gehen von der Beobachtung aus, dass jeder aufgrund des menschlichen Körperbaus mit der Anlage seiner Gliedmaßen und Sinnesorgane einen kleinen, nach vorne orientierten Raum besitzt, der für die Manipulation von Gegenständen und für Wahrnehmungen besonders geeignet ist. Dieser Raum ,gehört' ihm gewissermaßen, es ist sein „transactional segment“. Indem die Teilnehmer an fokussierten Interaktionen ihre Transaktionssegmente überlappen lassen, bilden sie eine sogenannte „F-formation“, deren Funktion es ist, allen Teilnehmern Zugang zur Interaktion zu gewähren (ausführlich: Kendon 1990c). Durch die F-Formation entsteht der sogenannte „o-space“ oder auch „interactional space“ (Ciolek/Kendon 1980: 243): der Raum, der von den Teilnehmern umschlossen wird, in den hinein die Stimmen projiziert werden, in den hinein gestikuliert wird, Hände geschüttelt werden usw. Der o-space zeigt durch die spezifische Ausprägung seiner F-Formation die Art der ablaufenden Interaktion an: Die Kontextanalyse hat hier eine Reihe unterschiedlicher F-Formationen beschrieben, die sie nach ihrer Ähnlichkeit zu lateinischen Großbuchstaben als N-, H-, V- (,geschlossene‘ Formationen) bzw. L-, C- oder I-Räume (,offene‘ Formationen) benennt. ${ }^{8}$ Der o-space ist nicht hermetisch nach außen abgeschlossen, sondern gliedert die räumliche Umwelt in einen gestufte Folge weiterer dynamisch hergestellter Räume („domains“): etwa den „p-space“, in dem sich die Teilnehmer bewegen, den „r-space“, der sich zwar hinter den Teilnehmern befindet, ihnen aber immer noch zugehörig ist (sodass jemand, der sich durch diesen Raum hindurch bewegt, das anzeigen muss), der „C-space“, der von den Teilnehmern überwacht wird, etwa um zu sehen, wer ihre Formation ,bedroht", oder schließlich der „b-space“, der öffentliche Raum, der keinem gehört.

8 In der Konversationsanalyse sind heute Bezeichnungen verbreiteter, die die Orientierung der Interaktionsbeteiligten zueinander alltagssprachlich beschreiben, etwa „face-to-face“, „side-byside" usw. 
Die Erkenntnisse der Kontextanalyse sind - mit Teilen ihrer Terminologie von der konversationsanalytischen Forschung zum Interaktionsraum rezipiert worden, gleichzeitig aber auch eingebettet worden in genuin konversationsanalytische Forschungsthemen, etwa das der Emergenz der Ordnung und der Sequenzialität der Interaktion (s. etwa Mondada 2007 oder 2009a).

Mondada (2007) beispielsweise zeigt, wie Raum-Konstitutionsaktivitäten in übergeordnete gemeinsame Aktivitäten (konkret: Wegbeschreibungen) eingebettet sind. Die Autorin zeigt, wie die Interaktionsbeteiligten nach der Ankündigung der Beschreibungsaktivität die Wegerklärung genau solange aufschieben, bis sie mit ihren Körpern eine Position eingenommen haben, die für die Wegbeschreibung nützlich ist. Die Herstellung des Interaktionsraums vollzieht sich in einem Einschub, der strukturell einer Verständigung sichernden Nebensequenz gleicht (Jefferson 1972), sie ist also ,eingebaut‘ in die Sequenzialität der übergeordneten Aktivität.

Untersucht wurde der insbesondere der Aufbau des Interaktionsraums in der Anfangsphase sozialer Interaktion (s. beispielsweise Mondada 2009, Mondada/Schmitt 2010, Mortensen/Hazel 2014), aber auch Momente der Umstruktuierung von Interaktionsräumen beim Übergang von einer Aktivität zu einer anderen (etwa Müller/Bohle 2007 am Beispiel von Instruktionssequenzen in Tangostunden oder Stefani 2013 zu räumlichen Folgen der aktiven Beteiligung von Geführten an Stadtführungen). Dabei gerät auch die innere Struktur des Interaktionsraums in den Blick. Pitsch (2012) etwa bereichert das Konzept des Interaktionsraums durch die empirische Analyse unterschiedlicher und unterschiedlich intensiver Beteiligungsformen und deren Auswirkungen auf eine vielfältige interne Strukturierung des Interaktionsraums. Die Komplexität des Interaktionsraums nimmt zu, sobald die Anzahl der Interaktionspartner zunimmt, aber auch sobald die Interaktionsbeteiligten sich durch den Raum bewegen (s. etwa Mondada 2016; zu mobilen Interaktionsräumen s.u., 5.2.).

Eine Schärfung des Interaktionsraum-Konzepts, die für die folgende Analyse besonders relevant ist, bietet Hausendorfs Konzept der „Situierung“ als einer kommunikativen Aufgabe (Hausendorf 2010). Indem die Interaktionsbeteiligten die Aufgabe der Situierung bearbeiten, stellen sie ihre gemeinsame Anwesenheit in der Interaktionssituation (als Grundbedingung der face-to-face-Kommunikation) her und verankern ihre Interaktion in der räumlichen Umwelt. Diese Verankerung betrifft nun, wie Hausendorf/Schmitt (2016: 35) schreiben, „nicht nur eine ,Sprech- und Zuhörsituation', sondern immer auch eine Wahrnehmungs-, Bewegungs- und Handlungssituation“. Deshalb lässt sich die Aufgabe der Situierung in drei Teilaufgaben untergliedern, durch die die Interaktionspartner je einen von drei „Sub-Räumen“ hervorbringen. 
- Der „Wahrnehmungsraum“ wird durch die Teilaufgabe der „Koorientierung“ hergestellt: Die Interaktionsteilnehmer sorgen für eine gemeinsame Aufmerksamkeitsausrichtung.

- Der „Bewegungsraum“ wird durch die Teilaufgabe der „Koordinierung“ erzeugt: Die Interaktionspartner stimmen ihre Bewegungen durch den Raum so aufeinander ab, dass sie als Formation zusammenbleiben.

- Der „Handlungsraum“ ergibt sich schließlich aus der Bearbeitung der Teilaufgabe der „Kooperation“. 9

Die Rekonstruktion der drei Teilaufgaben der Situierung gestattet eine feinere Einsicht in die Mechanik der Raumkonstruktion in der Interaktion. Bei meiner Analyse der Herstellung des für den Museumsbesuch spezifischen Interaktionsraums in Kapitel 5 werde ich daher auf Hausendorfs Konzeptionalisierung des Interaktionsraums zurückgreifen und ausgehend davon den musealen Bewegungs-, Wahrnehmungs- und Handlungsraum rekonstruieren. Anders als in der oben referierten Interaktionsraumforschung steht dabei die Frage im Mittelpunkt, wie genau die konkrete Ausgestaltung des Interaktionsraums mit der sozialen Praxis des gemeinsamen Museumsbesuchs verbunden ist. Ich werde zeigen, dass es die musterhafte Ausprägung der drei Subräume und ihrer Überschneidungen ist, die die gemeinsame Aktivität der Interaktionsbeteiligten wiedererkennbar zu einem Fall der sozialen Praxis „gemeinsamer Museumsbesuch“ werden lässt. Mit der Rekonstruktion der Musterhaftigkeit des musealen Interaktionsraums wird somit, wie ich belegen werde, ein zentraler Aspekt dieser sozialen Praxis erfasst.

\subsubsection{Die Multimodalität der Raumherstellung}

Die Aktivitäten der Raumkonstruktion oder "Situierung“, die in den bisher erwähnten Forschungsarbeiten beschrieben worden sind, greifen ausnahmslos auf mehr als einen Modus zurück: auf die Positionierung des Körpers im Raum, Blicke, Gesten, Sprache usw. Das bedeutet, dass bei der Raumkonstruktion nicht nur die Konstruktionsaktivitäten der einzelnen Interaktionsteilnehmer aufeinander abgestimmt werden müssen, sondern auch, wie die Konstruktionsbeiträge

9 Hausendorf (2010) nimmt noch einen vierten Sub-Raum an, den „Spielraum“, der dann zum Tragen kommt, wenn Interaktionsbeteiligte Räume nicht nur benutzen, sondern mit Rückgriff auf Konventionen ,lesen` und verstehen. Der Aspekt der Konventionalität scheint mir aber in allen Räumen eine Rolle zu spielen, wie die Analysen in Kapitel 5 zeigen werden. In späteren Arbeiten von Hausendorf ist der „Spielraum“ nicht mehr enthalten. 
der einzelnen Modi in der Interaktion miteinander verzahnt werden. Dieser Frage widmen sich eine Reihe von Arbeiten aus der linguistischen Gesprächsanalyse unter dem Etikett der „Koordination“ (s. den Sammelband Schmitt 2007). „Koordination" meint

solche Verhaltensweisen und -aspekte, die im Zusammenhang und zeitgleich mit verbalen Kooperationsbeiträgen und als deren Voraussetzung in den unterschiedlichen Modi körperlichen Ausdrucks realisiert werden, die jedoch selbst nicht als zielorientierte handlungsschematisch bezogene Beiträge angesehen werden. (Deppermann/Schmitt 2007: 23)

Grundsätzlich werden „intrapersonelle“ und „interpersonelle“ Koordination unterschieden. Mit intrapersoneller Koordination ist ein Aspekt der Selbstorganisation gemeint, mit denen Individuen den Einsatz mehrerer Modi aufeinander abstimmen, mit denen sie sich an der Interaktion beteiligen (2007: 32). Durch interpersonelle Koordination hingegen richten Interaktionsbeteiligte ihre multimodalen Beiträge auf ihre Interaktionspartner und deren Aktivitäten, Wahrnehmungsmöglichkeiten, Beteiligungspotenziale usw. aus. Krafft/DausendschönGay (2007) zeigen anhand der detaillierten Analyse einer kurzen Szene, die während eines Umzugs aufgenommen worden ist, dass interpersonale Koordination zum einen „direkt“ erfolgen kann, also z.B. indem ein Blickkontakt zwischen Interaktionsbeteiligten hergestellt wird, aber auch indirekt (ebd.: 173), indem der Blick eines anderen auf bestimmte, für das gemeinsame Handeln relevante Objekte gelenkt wird (s.a. Stukenbrock 2018 zum „Gesten-Blick-Nexus“).

Mit Hinblick auf die Frage, wie die Koordination unterschiedlicher Modi $\mathrm{zu}$ untersuchen ist, lassen sich zwei prinzipielle Vorgehensweisen unterscheiden. In der - von (Deppermann/Schmitt 2007: 38) so genannten „mikrophänomenologische[n] Perspektive“ werden die einzelnen Modi zunächst getrennt voneinander untersucht, um dann, quasi in einem zweiten Analysegang, nach den Mechanismen und Effekten ihrer Koordination zu fragen. Das entspricht im Wesentlichen dem Vorgehen der in Kapitel 4 referierten Text-BildForschung. Obwohl das Argument sicher gültig ist, dass durch das Weglassen eines Modus ein Verfremdungseffekt eintritt, der die Analysierenden Phänomene entdecken lässt, die unter unser alltäglichen Vertrautheit mit multimodaler Interaktion ,verschüttet ' sind, gelten dennoch die in Kapitel 4 angebrachten kritischen Punkte: die künstliche Festlegung relevanter Modi einerseits und, für die Konversationsanalyse mindestens ebensowichtig, das Auseinanderreißen multimodaler ,Gestalten' (Mondada 2014: 139). Durchgesetzt hat sich deshalb in der aktuellen Multimodalitätsforschung die Orientierung am „handlungsschematischen Zusammenhang“ (vgl. Deppermann/Schmitt 2007: 38f.), der auch das folgende Analysekapitel folgen wird. 


\subsubsection{Das Hineingebrachte und das Vorgefundene}

Die konversationsanalytische Forschung zum Interaktionsraum hat sich lange Zeit auf die Frage konzentriert, wie der Interaktionsraum mit Hilfe diverser Modi hergestellt wird, die die Interaktionsteilnehmer mit ihren Körpern in die Interaktionssituation hineinbringen. Die soziale Praxis des gemeinsamen Museumsbesuchs ist jedoch nicht vollständig erfasst, wenn man außer Acht lässt, was die Besucher im Ausstellungsraum vorfinden: den hochgradig semiotisch aufgeladenen Ausstellungsraum mit seinen Exponaten, Texten, Elementen von Ausstellungsdesign und Museumsarchitektur. ${ }^{10}$

Wie aber können Ausdrucksmittel in der Analyse zusammengebracht werden, die sich von ihrer Materialität und Zeitlichkeit stark voneinander unterscheiden? Während Körperbewegungen, Blicke, Zeigegesten und die gesprochene Sprache flüchtig sind, sind Bestandteile des umbauten Raums wie Mauern, Türen, Sitzmöbel, Beschilderungen usw. dauerhaft. Auch wenn sie sich von den Interaktionspartnern umgestalten lassen, sind dazu aber ganz andere, aufwändige Aktivitäten notwendig: ein Einreißen von Wänden mit dem Vorschlaghammer, ein Umstellen von Möbeln (Hausendorf 2012b), ein Zukleben oder Überkleben von Schildern usw. ${ }^{11}$

Ein Pionier der Erforschung des Zusammenhangs zwischen Interaktion und ihrer materiellen Umwelt ist Charles Goodwin (Goodwins erste Untersuchungen zu diesem Thema stammen aus den 1980er Jahren; siehe z.B. Goodwin 1986). Diesen Zusammenhang illustriert er etwa mit Hilfe der detaillierten Analyse eines Streits zwischen zwei Mädchen, die Himmel-und-Hölle spielen (Goodwin 2000). Das Spiel und der Streit der Mädchen (er dreht sich um die Frage, ob eine der beiden Spielerinnen in ein falsches Feld gesprungen ist) seien nicht $\mathrm{zu}$ verstehen, wenn man bei der Analyse nur die körpergebundenen (,embodied“) Modi berücksichtige, ohne auf die gemalten Linien am Boden Bezug zu nehmen:

10 Die Forderung, das in der Wahrnehmungssituation Vorgefundene bei der Analyse zu berücksichtigen, heißt nicht, von einer Determinierung des Verhaltens durch den Raum und die Exponate auszugehen (vgl. die Ausführungen hierzu in Heath/vom Lehn 2004, besonders S. 46-49). Ein Ansatz, der eine Zwischenposition einnimmt zwischen der Vorstellung einer Raumdeterminierung und derjenigen einer voraussetzungslosen interaktiven Herstellung des Raums und seiner Objekte, wird in Kapitel 6 entwickelt.

11 Diese Tatsache scheint mir Domke in ihrer Kommunikationstypologie mit dem Merkmal ,eingeschränkt generierbar“ erfassen zu wollen (Domke 2010a: 93). 
The actions that make up the game are impossible in a hypothetical «natural environment» unstructured by human practice, e.g. a field without the visible structure provided by the gridlines. (2000: 1505)

Gleiches gelte aber auch für die menschlichen Hervorbringungsaktivitäten:

[...] Simultaneously, the game is just as impossible without embodiment of the semiotic structure provided by the grid in a medium that can be actually jumped on. (2000: 1505)

Allgemein fordert er, „the social, cultural, material and sequential structure of the environment“ (2000: 1489) in die Analyse einzubeziehen. Die semiotischen Ressourcen der Umwelt sieht er in von ihm so genannte „graphic“ oder „semiotic fields“ organisiert, die mehrere Modi zugleich umfassen können. Mit den körpergebundenen Ausdrucksressourcen der Interaktionsteilnehmer sind sie über „contextual configurations“ verbunden, die Goodwin (2000: 1490) definiert als „,[a] particular, locally relevant array of semiotic fields that participants demonstrably orient to." Welche Modi jeweils zu einer kontextuellen Konfiguration gehören, entscheidet sich von Moment zu Moment in Abhängigkeit von der jeweils vollzogenen Handlung:

Contextual fields [...] are being continuously reshaped in order to accomplish relevant action. The range of phenomena that can be seen and evaluated changes at each step in this process. (2000: 1515$)$

Für die hier vorgestellte Untersuchung ist die Idee der „contextual configurations“ deshalb von Bedeutung, weil sie es erlaubt, ,Hineingebrachtes“ und ,Vorgefundenes in einem einzigen Analysegang zu untersuchen, und zwar „,as integrated components of a common process for the social production of meaning and action“ (2000: 1490).

Die Frage, wie die „material structure in the surround“ (2000: 1489) angemessen in eine konversationsanalytisch inspirierte Analyse von Interaktion einzubeziehen ist, liegt auch dem Begriffspaar „Interaktionsarchitektur“ und „Sozialtopographie“ zugrunde (s. die Arbeiten in Hausendorf/Schmitt/Kesselheim 2016, insbesondere Hausendorf/Schmitt 2016), mit dessen Hilfe untersucht werden soll, wie Interaktionsbeteiligte an Handlungspotenzialen in ihrer räumlichen Umgebung anknüpfen, wenn sie Elemente dieser Umgebung für ihre Interaktion relevantsetzen. Den Begriff „Interaktionsarchitektur“ erläutern Hausendorf/Schmitt (2016: 27) so:

Interaktionsarchitektur steht dabei für die Frage, wie die Architektur von Räumen Interaktion (wenn auch nicht determinieren und verhindern, so doch) ermöglichen und nahelegen 
kann und wie man diese interaktionsarchitektonischen Implikationen empirisch rekonstruieren kann.

Mit den „interaktionsarchitektonischen Implikationen“ sind Potenziale der Raumnutzung gemeint, die sich „sehr weitgehend an der sensomotorischen Ausstattung des Menschen festmachen lassen“ (2016: 44), etwa „Sichtbarkeit, Hörbarkeit, Be-Greifbarkeit, Begehbarkeit, Betretbarkeit, Verweilbarkeit oder Be-Handelbarkeit“ (2016: 36). Diejenigen Implikationen dagegen, die „stark von den sozial-räumlichen Wissensgrundlagen Anwesender abhängig sind“ (2016: 48) fassen die Autoren mit dem Begriff der „Sozialtopografie“ und betonen den engen Zusammenhang zwischen den mit dem Begriff der Sozialtopografie erfassten Erscheinungsformen und der Verankerung von Interaktion in institutionellen Kontexten („Rahmung“, 2016: 43f.). Die vorliegende Untersuchung der Kommunikation in der Ausstellung knüpft hier an, nutzt für die Analyse aber das Konzept der „Benutzbarkeitshinweise“, das keine Grenzlinie zwischen basal-anthropologischen und kulturell vermittelten Raumpotenzialen zieht, und nutzt zu deren Rekonstruktion Videos, die Raumnutzung in Interaktion dokumentieren (s. ausführlich Kapitel 6).

Aus der Perspektive der Interaktionsarchitekturanalyse sind eine ganze Reihe unterschiedlicher Räume zusammen mit der dort stattfindenden Interaktion untersucht worden, die unterschiedlich stark ,semiotisch aufgeladen' sind (Jucker et al. 2018): Unterrichtsraum (Putzier 2012), Hörsaal (Hausendorf 2012a, 2020), Kirche (Hausendorf 2016), Schalterraum (Hausendorf/Mondada 2013), öffentlicher Platz (in Jucker et al. 2018) oder die Sitzgruppe (Linke 2012). Untersucht wurden auch komplexe Räume, in denen die Raumkonstitution nicht nur durch die Bedingungen des menschlichen Wahrnehmungs- oder Bewegungsapparats beeinflusst ist, sondern von den Möglichkeiten und Beschränkungen der Raumwahrnehmung und -konstitution, die durch Technik und die Verwendung von Speicher- und Übertragungsmedien ins Spiel kommen. Beispiele hierfür sind Interaktionen auf Streamingplattformen (in Jucker et al. 2018) oder Mondada/ Oloff (2016), die untersuchen, wie Radiomoderatoren die Architektur und die Geräte des Radiostudios für ihre institutionellen Praktiken nutzen.

Hier berührt sich dieser Forschungsstrang mit den so genannten Workplace studies (s. etwa Drew/Heritage 1992, Suchman 1996, Heath/Luff 1996, Whalen/ Whalen/Henderson 2002, Whalen/Whalen 2004; vom Lehn 2018 für einen aktuellen Überblick), die über viele Jahrzehnte hinweg professionelles Handeln in verschiedenen, oft hochgradig strukturierten Arbeitsumgebungen erforscht haben: etwa in U-Bahn- oder Flughafenkontrollräumen, Callcentern usw. In all diesen Umgebungen, so die grundlegende Einsicht der Workplace studies, impliziere die Herstellung der Wirklichkeit 
engagement with the material features of settings; with technologies, artifacts, the physical configuration of buildings or other social spaces, and the like. (Whalen/Whalen 2004: 208)

Die Workplace studies sind schließlich auch deshalb für die Untersuchung der sozialen Praxis des gemeinsamen Museumsbesuchs interessant, weil das professionelle Handeln häufig nicht nur durch einen spezifisch interpretierten oder genutzten Raum bestimmt wird, sondern auch durch die Anwesenheit von und den Umgang mit speziellen Objekten, die hergestellt, manipuliert oder als Werkzeuge genutzt werden (s. die obigen Literaturangaben). $\mathrm{Zu}$ einer vollständigen Beschreibung der sozialen Praxis des gemeinsamen Museumsbesuchs gehört nämlich zweifellos auch die Beantwortung der Frage, wie die Museumsbesucher mit den Objekten im Ausstellungsraum, den Exponaten, umgehen. Außerhalb dieses Forschungsstrangs wurde die Rolle von Objekten in der Interaktion aus einer Reihe unterschiedlicher Perspektiven untersucht. ${ }^{12}$

- So zeigen Deppermann/Schmitt (2007) wie „signifikative Objekte“ (konkret: ein Monitor im Verlauf eines Filmdrehs) eine interaktionsstrukturierende Funktion haben können, Putzier (2012) zeichnet nach, wie ein Erlenmeyerkolben in einer Chemiestunde zum Mittelpunkt eines vom Lehrer mit einigem Aufwand hervorgebrachten „Demonstrationsraums“ wird, auf den sich die Aufmerksamkeit der Schüler konzentrieren soll und Mortensen/Hazel (2014) rekonstruieren, wie Objekte zur Verankerung von Interaktion in einem institutionellen Rahmen genutzt werden können.

- Erforscht wurde auch, wie Objekte im Lauf einer Interaktion Schritt für Schritt hergestellt werden (etwa Mitschriften im Lauf eines Unterrichtsgesprächs, Pitsch 2007) oder wie Objekte innerhalb von interaktiven Designprozessen entworfen werden (Murphy 2005, Pitsch/Krafft 2010).

- Unterschiedliche Grade des Einbezugs von Objekten in die Interaktion rekonstruieren Böhringer/Wolff (2010) am Beispiel der Nutzung des Computers in Beratungsgesprächen: Dieser Einbezug kann von einem vollständigen Ignorieren über den Status als einem ,Mitspieler' in der Interaktion bis hin zur Zuweisung einer zentralen Interaktionsrolle reichen.

Die Zahl der hier erwähnten Arbeiten, die die Rolle der räumlichen Umwelt und ihrer Objekte für die Interaktion untersuchen, darf nicht darüber hinwegtäuschen, dass der Einbezug der statischen, nicht an den Körper der Interaktionsteilnehmer gebundenen Modi in die Dynamik der Interaktion weiterhin ein Problem ist. Gerade wenn man sich für das,Vorgefundene‘ interessiert, also Eigenschaften

12 Einen guten Einblick in diesen Forschungsstrang gibt der Sammelband Nevile et al. (2014). 
des Raums oder der Objekte, die der untersuchten Interaktion vorausgehen, stellt das letzten Endes die Überzeugung vom prinzipiellen Herstellungscharakter der Wirklichkeit infrage, eine der Grundüberzeugungen der Konversationsanalyse. So schreibt die Designtheoretikerin Ackermann mit Hinblick auf die Bedeutung von Objekten:

People read into artifacts because of who they are, and because artifacts offer clues. Like archeological sites or eroded landscapes, they are marked by their history, and they embody the knowledge or collective experience that went into their being (both reflecting and transcending builders' and readers' intents and aspirations). The constructivist's nightmare may well come true! Yes, human-made artifacts can call upon certain experiences and uses, and discourage others. (Ackermann 2007: 7)

Ein Weg, das Vorgefundene in die Analyse der Kommunikation in der Ausstellung einbeziehen $\mathrm{zu}$ können, ohne die konstruktivistische Grundüberzeugung der Konversationsanalyse aufgeben zu müssen, wird in Kapitel 6 entwickelt werden, aufbauend auf den Analysen der Kommunikation in der Ausstellung in Kapitel 5.

\subsubsection{Wissenskommunikation}

Die Beschäftigung mit und die Erarbeitung von neuem Wissen ist in der Interaktion der Besucher in den von mir untersuchten Museen eine zentrale Orientierungsgröße. Es ist deshalb lohnend, die Kommunikation in der Ausstellung als Fall von Wissenskommunikation zu untersuchen.

Die Untersuchung der Rolle, die Wissen in Interaktion spielt, ist laut Deppermann (2018: 133) ,in den vergangenen zehn Jahren zu einem der am intensivsten erforschten Bereiche der Interaktionsanalyse geworden." Wissen kann in vielerlei Gestalt im Gespräch zutage treten, etwa in Form von ausdrücklichen Formulierungen eines Wissens oder Nichtwissens (in Aussagen wie ,Ich weiß nicht‘, Mondada 2011a), durch die Verwendung von Wörtern aus der Wortfamilie des Wissens (,wissen“, ,vermuten“ usw.), durch bestimmte Modalpartikeln wie ja, halt oder doch usw. In all diesen Fällen wird signalisiert, ob eine Aussage als Teil des geteilten Wissens verstanden werden soll, oder nicht („epistemic stance“, Heritage 2012). Darüber hinaus ist in der Konversationsanalyse intensiv untersucht worden, wie - in Worten von Heritage - der „epistemic status“ markiert wird, den ein Sprecher relativ zu seinem Interaktionspartner einnimmt, also ob er im Hinblick auf den relevanten Wissensbestand mehr weiß als jener (,K+“) oder weniger (,,K-“). Besonders interessant ist hierbei die Frage, wie der epistemische Status mit der Zuordnung von Interaktionsbeteiligten zu sozialen Kategorien verbunden ist (z.B. mit der Identifikation als Experte) und wie der epistemische 
Status und bestimmte Berechtigungen oder Verpflichtungen zusammenhängen (Stivers/Mondada/Steensing 2011). Darüber hinaus ist für die Analyse der Kommunikation in der Ausstellung auch die Orientierung der Sprecher an der Allgemeingültigkeit oder Regelhaftigkeit des Ausgesagten von Interesse, wenn man nachzeichnen möchte, wie Besucher ausgehend von ihrer Betrachtung der Exponate Wissen im Sinne von geteilten Gewissheiten über Sachverhalte konstruieren, die über den beobachteten Einzelfall hinausreichen. Und schließlich liefern auch die Bezugnahme auf fachliche Institutionen und Autoritäten oder die Nutzung fachsprachlicher Begriffe einen Hinweis darauf, dass wir es mit Wissenskommunikation zu tun haben: Das Wissen, um das es geht, wird dann als Wissen einer wissenschaftlichen Disziplin oder des gesellschaftlichen Funktionsbereichs Wissenschaft allgemein ausgewiesen, also als Fachwissen. (Dabei kommt es, wie wir sehen werden, nicht darauf an, ob dieses Wissen besonders komplex ist oder nur durch ein Studium erworben werden kann.)

„Wissen“ wird in dieser Arbeit nun nicht als individualpsychologisches Konzept untersucht. Vielmehr ist das hier vertretene Wissenskonzept ein in der Interaktion begründetes. Insbesondere stütze ich mich auf das ,interaktiv fundierte Wissenskonzept ${ }^{\star}$, wie es in Bergmann/Quasthoff (2010) entwickelt und begründet wird. Ausgangspunkt ist die Überzeugung, dass sich Wissen, verstanden als individuelle mentale Repräsentationen, nicht direkt beobachten lässt. Dieses prinzipielle Problem der „Unbeobachtbarkeit von Wissen“ (2010: 23-27) stellt sich nicht nur der Wissenschaft, beispielsweise wenn sie Lernprozesse im Museum untersuchen möchte (s.o. 4.1), sondern auch den Teilnehmern an alltäglicher Interaktion, also z.B. den Besuchern bei ihrem gemeinsamen Museumsbesuch. Was gewusst, was gelernt wird, ist immer nur dem betreffenden Individuum zugänglich (zumindest, soweit es sich um diskursives, also der sprachlichen Formulierung zugängliches Wissen handelt). Um dieses Problem zu überwinden, haben die Interaktionsteilnehmer Alltagsmethoden entwickelt, mit denen sie Wissen „aus seiner bloß subjektiven Gegebenheitsweise [...] befreien und zu einem Objekt intersubjektiver Verständigung und geteilter Sinngebung“ machen können (Bermann/Quasthoff 2010: 26). Diese Methoden nun führen zu (wie wir sehen werden: sprachlichem und körperlichem) Verhalten, das nicht nur für die Gesprächspartner zugänglich ist, sondern auch für die wissenschaftliche Analyse. Damit ist Wissen für die Gesprächsanalyse als kommunikativer Gegenstand greifbar, als eine im Gespräch gemeinsam etablierte und als geteilt signalisierte Überzeugung vom allgemeinen Zutreffen bestimmter Sachverhalte im Sinne eines „common ground“ (s. Clark 1996: 92-121, vgl. auch DausendschönGay/Domke/Ohlhus 2010b).

Die konversationsanalytische Perspektive auf Wissen zeichnet sich dadurch aus, dass nach den interaktiven Praktiken gefragt wird, mit denen Zugang zu 
Wissen im Verlauf der Interaktion behauptet oder bestritten, präzisiert, abgestützt und als geteilt erarbeitet wird. Mit der Konzentration auf die Methoden, mit denen die Interaktionsbeteiligten Wissen erarbeiten, geraten also der Prozess der Herstellung von Wissen und seine sprachlichen und nicht-sprachlichen Verfahren in den Blick: die „Aktualgenese“ von Wissen (Bergmann/Quasthoff 2010: 27). Anstatt nur auf das Produkt der Wissenskommunikation zu schauen - den Wissenszuwachs, das Gelernte - macht es das interaktive Wissenskonzept möglich zu fragen, wie genau die Interaktionsbeteiligten sich Schritt für Schritt dasjenige erarbeiten, was es für sie in der Ausstellung zu lernen gibt, und wie sie dieses neue geteilte Wissen mit ihrem Vorwissen in Beziehung setzen. Konzentriert man sich auf die Aktualgenese von Wissen, erweist sich die Wissenskonstruktion durch die Museumsbesucher als grundsätzlich interaktiv. Jedes Mitglied der Besuchergruppe kann jederzeit in diesen Prozess eingreifen, etwa indem es die Beiträge der anderen kommentiert, korrigiert oder zum Anlass für einen eigenen Beitrag nimmt. Der Prozess und das Ergebnis der Wissenskonstruktion ist daher immer dem ,Interaktionsteam‘ als Ganzem zuzuschreiben, selbst wenn einzelne Mitglieder weniger aktiv oder weniger offensichtlich an der Konstruktion eines bestimmten Wissenselements teilgenommen haben. Und die Wissenskonstruktion erweist sich als strikt lokal. Mit anderen Worten: Sie entsteht sukzessive im Verlauf der Interaktion, und jeder Beitrag muss vor dem Hintergrund seiner vorausgehenden und im Hinblick auf seine Konsequenzen für die folgenden Beiträge gesehen werden. Um den Prozess der Wissenskonstruktion beim Museumsbesuch zu rekonstruieren, reicht es deshalb nicht, eine Liste der erarbeiteten Wissensbestände zu erstellen. Vielmehr geht es darum herauszufinden, mit welchen Schritten und unter Hinzuziehung welcher kommunikativer Verfahren ein bestimmter Sachverhalt als geteiltes, auf ein wissenschaftliches Fach bezogenes Wissen ausgehandelt wird.

Ist aber das geteilte Wissen, das in der Kommunikation gemeinsam konstruiert wird, wirklich „Wissen“? Anders gesagt: Was ist, wenn sich die von den Besuchern erarbeiteten gemeinsamen Überzeugungen von dem unterscheiden, was die Wissenschaft herausgefunden hat und als „Wissen“ bezeichnet? Der Abgleich des lokal konstruierten Wissens mit dem wissenschaftlichen Fachwissen scheint mir nur aus einer angewandten Perspektive nützlich zu sein. Dann also, wenn man wissen möchte, ob die Besucher das gelernt haben, was sich die Kuratoren von dem Arrangement in der Vitrine oder dem Ausstellungsraum erhofft haben. Mich dagegen interessiert die grundlegende, Mechanik ${ }^{`}$ der Wissenskonstruktion beim gemeinsamen Museumsbesuch. Ich möchte wissen, welche kommunikativen Verfahren die Besucher anwenden, wenn sie das komplexe multimodale Arrangement im Ausstellungsraum nutzen, um sich Fachwissen zu erschließen. Dazu gehört unter Anderem, 
- wie sie die Elemente der räumlichen Umwelt als ,Wissensdinge“ adressieren, also als bedeutungsvolle Objekte, aus deren Betrachtung sich etwas lernen lässt (vgl. Niewerth 2018: 45-48 zu Museumsobjekten als „epistemischen Dingen“);

- wie sie sich gegenseitig aufzeigen, dass ihre Beobachtungen und Behauptungen sich auf wissenschaftliche Erkenntnisse, Aktivitäten oder Akteure beziehen; oder

- wie sie spezifische Instanzen zur Stützung ihres behaupteten Wissens heranziehen (bspw. Ausstellungstexte, eigene Beobachtungen oder Verweise auf wissenschaftliche Bücher oder Sendungen).

Das interaktionale Wissenskonzept ermöglicht einen fruchtbaren Einblick in einen zentralen Bestandteil der sozialen Praxis des gemeinsamen Museumsbesuchs. Es erlaubt, die Verfahren zu rekonstruieren, mit denen die Besucher beim gemeinsamen Gang durch das Museum den Ausstellungsraum als „Lernort“ benutzen. Es erlaubt, das beobachtete sprachliche und körperliche Verhalten der Besucher mit den Bedeutungspotenzialen im Ausstellungsraum in Beziehung setzen, die in Kapitel 3 rekonstruiert worden sind, und zu beschreiben, wie die Wissenskonstruktion der Besucher an diesen Potenzialen anknüpft (oder sich über sie hinwegsetzt). Diese substanziellen Einsichten lassen sich gewinnen, ohne von der Annahme ausgehen zu müssen, dass sich aus den Gesprächsdaten direkt auf das Vorliegen bestimmter mentaler Repräsentationen oder Prozesse der Museumsbesucher schließen ließe. 